\title{
DISTÚRBIOS PSIQUIÁTRICOS RELACIONADOS AO ÁLCOOL ASSOCIADOS A DIAGNÓSTICOS DE CLÍNICA MÉDICA E/OU INTERVENÇÕES CIRÚRGICAS, ATENDIDOS NUM HOSPITAL GERAL
}

\author{
Eliene Reis de Oliveira* \\ Margarita Antonia Villar Luis**
}

OLIVEIRA.,E.R.; LUIS.,M.A.V. Distúrbios psiquiátricos relacionados ao álcool associados a diagnósticos de clínica médica e/ou intervenções cirúrgicas, atendidos num hospital geral. Rev.latino-am.enfermagem, Ribeirão Preto, v. 5, número especial, p. 51-57, maio 1997.

Através de um inquérito epidemiológico, buscou-se detectar as co-morbidades dos diagnósticos psiquiátricos relacionados ao álcool. Para tanto, investigou-se os atendimentos num Hospital de Urgências Médicas no Setor de Psiquiatria, no período de 1988 a 1991, tendo por objetivo identificar quais as doenças clínicas e outros incidentes que acometem a população referida. Fizeram parte do levantamento os diagnóstico relacionados ao álcool, conforme CID 9 (291; 303 e 305.0) e os associados não pertencentes à psiquiatria. Evidenciou-se no período, 1835 casos com diagnósticos relacionados ao álcool e destes 487 (26.5\%) se referiram aos grupos 291-303-305.0, associados à outros não psiquiátricos. Desse total a maior frequência de casos ocorreu nos grupos 340-349 (Outros transtornos do SNC) e 800-859 (Lesões e complicações traumáticas) com $80(16.4 \%)$ atendimentos, respectivamente. Observando-se os dados por ano, constata-se que dos diagnósticos relacionados ao álcool (com diagnósticos clínicos associados) o 303 (Síndrome de Dependência Alcoólica) foi o que teve maior número de atendimento com 326 (67\%) casos, seguido de 291 (Psicose Alcoólica) com 117 (24\%) e 305.0 (Álcool) com 44 (9\%). No período, tanto o diagnóstico psiquiátrico 303 como o 291, apareceram mais frequentemente associados ao grupo de diagnóstico clínicos 340-349, com 55 (11.2\%) e 12 (2.4\%) casos, respectivamente e o 305.0 teve o grupo 800-859 com 10 (2\%) casos, como o mais frequente.

UNITERMOS: alcoolismo, clínica médica e álcool, intervenções cirúrgicas e álcool, psiquiatria

\section{INTRODUÇÃO}

Os estudiosos em Saúde Mental são unânimes em afirmar que o alcoolismo vem alcançando proporções crescentes entre a população, o que pode ser verificado no número de internações psiquiátricas onde esse diagnóstico tem estado entre os principais, quando não o primeiro entre as internações e reinternações psiquiátricas (CVE, 1991).

Contudo, o paciente alcoolista também está presente nos serviços de Pronto Atendimento médico, fato frequentemente observado pelos profissionais de saúde, embora não se tenham encontrado estudos relatando essa frequência.

O diagnóstico de alcoolismo tem sido definido com base em quatro grupos de indicadores: consumo, dependência psicológica, dependência física e problemas relacionados ao álcool (SCHUCKT,1985).
Hill apud EDWARDS (1985) dizia, em meados da década de 80, não existirem evidências do alcoolismo ser ou não uma doença. A ausência de um padrão homogêneo, de um curso único, ao contrário do que se pensava antes, faz com que alguns prefiram ver o alcoolismo como uma síndrome e não como uma doença.

O alcoolismo é visto como uma toxicomania pela OMS (WHO, 1970), que a conceitua como "um estado psíquico e algumas vezes também físico, resultante da interação entre o organismo vivo e uma substância, caracterizado por um comportamento e outras reações que incluem sempre compulsão para ingerir a droga, de forma contínua ou periódica, com a finalidade de experimentar seus efeitos psíquicos e às vezes para evitar o desconforto de sua abstinência. A tolerância pode existir ou faltar e o indivíduo pode ser dependente de mais de uma droga".

Essa conceituação refere-se à toxicomania ou farmacodependência, nesse caso o alcoolismo fica caracterizado como tal, devido a que o alcoólatra tende a

\footnotetext{
* Graduanda do curso de Enfermagem da Escola de Enfermagem de Ribeirão Preto-USP e bolsista de Iniciação Científica

** Prof $^{a}$ Dr $^{\text {a }}$. do Departamento de Enfermagem Psiquiátrica e Ciências Humanas da Escola de Enfermagem de Ribeirão Preto-USP, orientadora do trabalho

Financiamento do Conselho Nacional de Pesquisa e Tecnologia - CNPq
} 
aumentar progressivamente as doses ingeridas de tal álcool e quando interrompe completa ou bruscamente a ingestão do mesmo, apresenta um grave conjunto de sinais e sintomas físico-psíquicos, que caracterizam a Síndrome de Abstinência Alcoólica.

O alcoolismo, segundo a OMS, é uma doença de natureza complexa, na qual o álcool atua como fator determinante sobre causas psicossomáticas preexistentes no indivíduo e para cujo tratamento é preciso recorrer a processos profiláticos e terapêuticos de grande amplitude. Já os alcoólatras são bebedores excessivos, cuja dependência do álcool chega a ponto de acarretar-lhes pertubações mentais evidentes, manifestações que afetam a saúde física e mental, suas reações individuais, seu comportamento socieconômico ou pródromos de perturbações desse gênero e que, por isso, necessitam de tratamento (CORDEIRO et al., 1954).

$\mathrm{Na}$ atualidade houve substituição desse termo, passando-se a denominar os usuários bebedoresproblemas e os dependentes de álcool em alcoolistas (BRASIL. M.S.,1990).

O termo alcoolismo foi empregado pela primeira vez em 1856, pelo médico sueco, Magnus-Huss, para designar os sinais e sintomas físico-psíquicos surgidos pela excessiva ingestão de doses elevadas (e por tempo prolongado) de bebidas alcoólicas (FORTES, 1975), Jellinek apud FORTES (1975), um dos mais brilhantes pesquisadores do "Yale Center of Alcohol Studies", define que o alcoolismo é qualquer uso de bebidas alcoólicas que ocasione prejuízo físico e/ou interpessoais e correlaciona a sua existência quando existe a perda da liberdade sobre o ato de beber.ao indivíduo, à sociedade ou a ambos.

MASUR (1984), tem a convicção de que o alcoolismo é uma doença na medida em que implica numa situação de dependência tão intensa, que implica em visíveis prejuízos a nível físico, psíquico e/ou interpessoal e correlaciona a sua existência quando existe, à perda da liberdade sobre o ato de beber.

Há grande diversidade de opiniões, observandose a existência de autores que admitem os sintomas da intoxicação crônica pelo álcool como sendo roupagens que escondem ou disfarçam uma personalidade mórbida. Não haveria, propriamente, alcoolismo primário, sendo os casos relatados como alcoolismo, secundários a "algo" subjacente à personalidade do paciente. Em outras palavras, o indivíduo normal nunca se tornaria um alcoolista crônico. Haveria sempre necessidade da existência de um fator ligado a uma personalidade mórbida (FORTES, 1975).

Contrários a esse ponto de vista, Mayer-Gross apud FORTES (1975) relata que nem todos os alcoólatras teriam personalidades fortemente predispostas e argumentam que a massa de alcoolistas não provém unicamente deste ou daquele grupo em particular, constituindo sempre um grupo muito heterogêneo.

MASUR (1984), no concernente a causa, refere não haver uma resposta breve, clara e convincente: existem várias tentativas de respostas como vulnerabilidade biológica, vulnerabilidade psicológica e vulnerabilidade social. Isto partindo da premissa de que todos os que bebem têm potencialmente a possibilidade de se tornarem alcoolistas. A maior ou menor probabilidade vai depender da interação entre os diferentes fatores da vulnerabilidade.

A título de conclusão, parece não haver uma causa única ou isolada, o beber pode resultar de uma variedade de inter-relações de influências, em função de que cada variante repercute de forma ímpar em cada pessoa (CERVESATO, 1993).

Embora não tenham sido feitos estudos, associando os quadros relacionados ao álcool com diagnósticos não psiquiátricos, essa associação é de conhecimento comum dos profissionais da área da saúde particularmente, médicos e enfermeiros. Contudo, parece não ter havido uma preocupação maior em desenvolver pesquisas relacionando danos psíquicos e físicos, advindos do hábito excessivo de beber.

Os dados do relatório sobre Internações Psiquiátricas no Estado de São Paulo (CVE, 1991) referentes ao período 1986-1988, são preocupantes no que diz respeito ao alcoolismo e psicose alcoólica. Nesse documento, esse grupo de diagnóstico aparece em terceiro lugar, no que se refere à frequência de óbitos no sexo masculino. Cabe ressaltar, que a idade média de óbitos, encontrada nessa população foi de 45 anos.

Dada a relevância do problema, o presente estudo tem o objetivo de identificar os diagnósticos relacionados ao álcool CID-9 - 291(Psicose Alcoólica); 303 (Síndrome de Dependência ao Álcool) e 305.0 (Álcool)), atendidos num Setor de Emergências Psiquiátricas de um Hospital Geral ligado à Universidade da cidade de Ribeirão Preto, que apresentaram associação com diagnósticos das clínicas médica e/ou cirúrgica, atendidos no período de 1988 a 1991, identificando os grupos de doenças clínicas e/ou incidentes que acometem essa população.

\section{METODOLOGIA}

Trata-se de um inquérito epidemiológico, retrospectivo, com informações oriundas do Setor de Processamento de Dados e Estatística de um Hospital Universitário da cidade de Ribeirão Preto-SP (HCRP). O material foi agrupado conforme diagnósticos relacionados ao álcool (CID-9); e como cada paciente apresentava mais de um diagnóstico, eles foram separados por grupos de diagnósticos. Foram utilizados como base, as categorias diagnósticas do CID-9. 


\section{RESULTADOS}

No período estudado encontrou-se 1835 atendimentos relacionados ao uso de álcool $(303,291$ e 305.0) e destes 487 atendimentos apareceram associados à outros diagnósticos não pertencentes à psiquiatria.

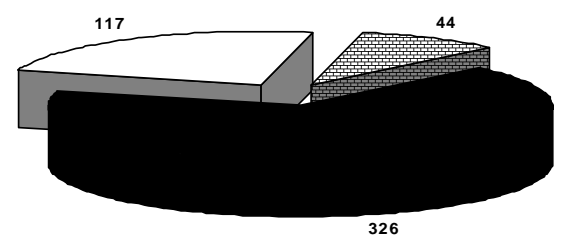

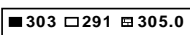

Figura 1 - Distribuição do total de diagnósticos (291, 303 e 305.0) atendidos no período 1988-1991 em Ribeirão Preto-SP

Conforme se observa na Figura 1, em relação aos diagnósticos não psiquiátricos, foram encontrados $326(67 \%)$ associados com o diagnóstico psiquiátrico 303 (Síndrome de Dependência Alcoólica); verifica-se ainda, a existência de 117 (24\%) associados ao 291 (Psicose Alcoólica); e 44 (9\%) associado ao 305.0 (Álcool sem Dependência).

Analisando-se a frequência dos diagnósticos relacionados ao álcool mais outros não psiquiátricos temse os seguintes dados expostos na figura 2.

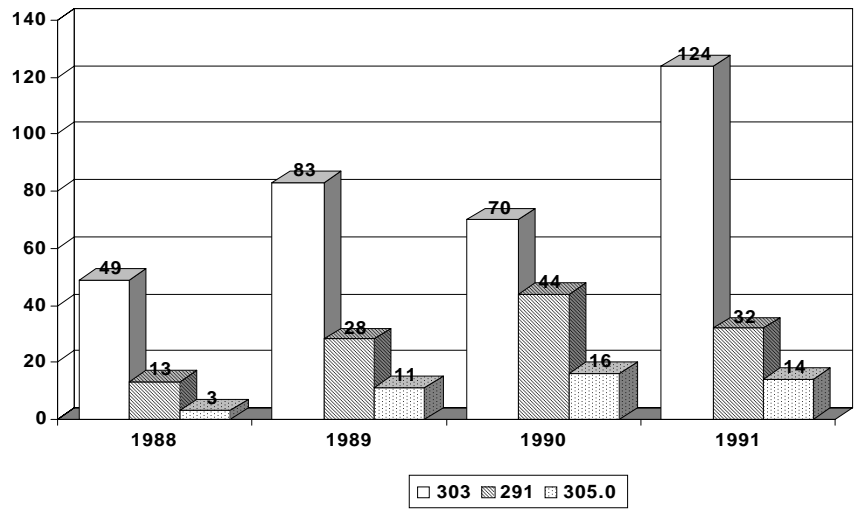

Figura 2 - Distribuição dos diagnósticos relacionados ao álcool associados à outros não pertencentes à psiquiatria, por ano em Ribeirão Preto-SP, de 1988 a 1991

$\mathrm{Na}$ Figura 2, pode ser constatado que o diagnóstico Síndrome de Dependência do Álcool (303) foi o que apareceu mais frequentemente associado a outros da clínica médica e cirúrgica, em todo o período com um total de $326(66,9 \%)$ casos, apresentando sua maior elevação nos anos de 1991 e 1989 respectivamente.
A Psicose Alcoólica apresenta-se como o segundo diagnóstico de maior ocorrência (embora com números bem inferiores aos do primeiro), em termos das mesmas associações, com 117 (24\%) tendo o seu pico em 1990 e depois em 1991. Como último diagnóstico em frequência, tem-se o álcool sem dependência (associado a outros diagnósticos não psiquiátricos), também apresentando número de casos bem reduzido, em relação aos dois primeiros, com 44 (9\%) caso; porém observa-se que ele vem aumentando no período (com declínio mínimo em 1991).

No que concerne aos diagnósticos não psiquiátricos, o Quadro 1 permite a visualização da distribuição das categorias diagnósticas que mais frequentemente foram associadas aos diagnósticos psiquiátricos relacionados ao álcool.

Quadro 1 - Distribuição das categorias diagnósticas relacionados à clínica médica e/ou intercorrências cirúrgicas mais frequentes atendidas no período 19881991 em Ribeirão Preto-SP

\begin{tabular}{|c|c|c|c|c|c|}
\hline CATEGORIAS & 1988 & 1989 & 1990 & 1991 & Total \\
\hline $\begin{array}{l}340-349-\text { Outros } \\
\text { transtornos do SNC } \\
\text { (epilepsia, esclerose } \\
\text { multipla e outros) }\end{array}$ & 4 & 22 & 30 & 28 & 84 \\
\hline $\begin{array}{l}800-959 \text { - Lesões e } \\
\text { complicações traumáticas } \\
\text { (fraturas, traumatismos, } \\
\text { ferimentos superficiais, } \\
\text { contusóes...) }\end{array}$ & 10 & 19 & 27 & 24 & 80 \\
\hline $\begin{array}{l}530-579 \text { - Doenças do } \\
\text { aparelho digestivo em geral } \\
\text { (esôfago, estômag, necrose, } \\
\text { abcessos e doenças crônicas } \\
\text { do figado e outros) }\end{array}$ & 8 & 23 & 15 & 27 & 73 \\
\hline $\begin{array}{l}960-989 \text { - Intoxicações } \\
\text { (drogas, medicaçóes e } \\
\text { outras) e efeitos tóxicos } \\
\text { (subst. de origem não } \\
\text { medicinal) }\end{array}$ & 11 & 10 & 16 & 19 & 56 \\
\hline $\begin{array}{l}\text { 780-789 - Sintomas, sinaise } \\
\text { afecçóes mal definidas } \\
\text { (SWC, pel, nutrição, cabeça, } \\
\text { aparelho urináno e outros) }\end{array}$ & 10 & 10 & 10 & 23 & 53 \\
\hline $\begin{array}{l}401-405 \text { - Doença } \\
\text { hipertensiva }\end{array}$ & 7 & 10 & 5 & 14 & 36 \\
\hline $\begin{array}{l}260-269 \text { - Deficiências } \\
\text { nutricionais }\end{array}$ & 6 & 9 & 8 & 9 & 32 \\
\hline 480-487-Pneumoria e gripe & - & 3 & 8 & 10 & 21 \\
\hline $\begin{array}{l}\text { Outros -Refere-se a } \\
\text { diagnósticos com números } \\
\text { inferiores a } 5\end{array}$ & 9 & 16 & 11 & 16 & 52 \\
\hline Total & 65 & 122 & 130 & 170 & 487 \\
\hline
\end{tabular}

* Houve pacientes que apresentaram até 3 diagnósticos, associados ao psiquiátrico 
Todos os diagnósticos psiquiátricos relacionados ao álcool apareceram mais frequentemente associados à categoria diagnostica Outros transtornos do SNC (340349), com 84 (17,2\%) casos no período, seguido de Lesões e complicações traumáticas (800-959) com 80 $(16,4 \%)$ casos e Doenças do aparelho digestivo em geral (530-579) com 73 (14,9\%) casos. As demais categorias diagnósticas apresentaram números inferiores a 60 casos, conforme observa-se no Quadro 1.

Constata-se, no que diz respeito às categorias de maior ocorrência de associações com diagnósticos relacionados ao álcool, por ano, o seguinte: no ano de
1988 predominaram às Intoxicações e efeitos tóxicos (960-989). No ano de 1989, aparecem como mais frequentes as Doenças do aparelho digestivo em geral (530-579) tais como as do esôfago, estômago, doenças crônicas do fígado e outras. Já nos anos de 1990 e 1991 a categoria diagnóstica dominante para ambos os anos refere-se a Outros transtornos do SNC (340349) como a epilepsia, esclerose múltipla.

No intuito de identificar quais as categorias diagnósticas orgânicas aparecem mais associadas com diagnósticos psiquiátricos relacionados ao álcool foi elaborado o Quadro 2.

QUADRO 2. Distribuição dos pacientes conforme categorias diagnósticas orgânicas associadas aos quadros relacionados ao álcool, no período 1988-1991 em Ribeirão Preto-SP

\begin{tabular}{|c|c|c|c|c|}
\hline CATEGORIAS & $\begin{array}{l}303 \\
\mathrm{n}^{\circ}\end{array}$ & $\begin{array}{c}291 \\
\mathrm{n}^{\circ}\end{array}$ & $\begin{array}{c}305.0 \\
\mathrm{n}^{\circ}\end{array}$ & Total \% \\
\hline $\begin{array}{l}340-349 \text { - Outros transtomos do SNC (epilepsia, esclerose } \\
\text { multipla e outros) }\end{array}$ & 61 & 19 & 4 & $84(17.2)$ \\
\hline $\begin{array}{l}880-959 \text { - Lesões e complicações traumáticas (fraturas, } \\
\text { traumatismos, ferimentos superficiais, contusóes...) }\end{array}$ & 47 & 18 & 15 & $80(16.4)$ \\
\hline $\begin{array}{l}530-579 \text { - Doenças do aparelho digestivo em geral } \\
\text { (e soffago, estômago, necrose, abcessos e doenças } \\
\text { crônicas do figado e outros) }\end{array}$ & 47 & 25 & 1 & $73(14.9)$ \\
\hline $\begin{array}{l}\text { 960-989 - Intoxicações (drogas, medicações e outras) e } \\
\text { efeitos tóxicos (subst. de origem não medicinal) }\end{array}$ & 36 & 5 & 15 & $56(11.5)$ \\
\hline $\begin{array}{l}\text { 780-789-Sintomas, sinais e afecções mal definidas (SNC, } \\
\text { pele, nutrição, cabeça, aparelho urinário e outros) }\end{array}$ & 36 & 13 & 4 & $53(10.8)$ \\
\hline 401-405 - Doença hipertensiva & 27 & 8 & 1 & $36(7.4)$ \\
\hline 260-269 - Deficiências nutricionais & 22 & 9 & 1 & $32(6.6)$ \\
\hline 480-487 - Pneumonia e gripe & 12 & 8 & 1 & $21(4.3)$ \\
\hline $\begin{array}{l}\text { Outros - Refere-se a diagnóstico com números inferiores } \\
\text { a } 5\end{array}$ & 38 & 12 & 2 & $52(10.6)$ \\
\hline Total & 326 & 117 & 44 & 487 \\
\hline
\end{tabular}

303 (Síndrome de Dependência Alcoólica)

291 (Psicose Alcoólica)

305.0 (Álcool sem Dependência)

O diagnóstico psiquiátrico Síndrome de Dependência do Álcool (303) apareceu mais associado à categoria diagnóstica Outros transtornos do SNC (340349) com $61(18,7 \%)$ casos, e em segundo lugar com o mesmo número de casos, Lesões e complicações traumáticas (800-859) e Doenças do aparelho digestivo em geral (530-579) ambos com 47 (14,4\%); já as categorias Intoxicações e efeitos tóxicos (960-989) e Sintomas, sinais e afecções mal definidas (780-789), apresentam-se com números iguais de $36(11,0)$ casos, ficando em terceiro lugar. A Psicose Alcoólica (291) esteve associado mais frequentemente, a Doenças do aparelho digestivo em geral (530-579) com 25 (21,3\%), seguido de Outros transtornos do SNC (340-349) com $19(16,2 \%)$ e Lesões e complicações traumáticas (800$859)$ com $18(15,3 \%)$ casos. Essa última categoria está relacionada a fraturas, traumatismos e outros quadros dessa natureza.

Cabe ressaltar que o diagnóstico Psicose Alcoólica (291) apresentou número de casos bem inferior 
(24\% do total) ao apresentado pela Síndrome de Dependência do Álcool (303) ( 66,9\% do total).

No referente ao diagnóstico Álcool sem dependência (305.0), este esteve, primeiramente mais associado às categorias Lesões e complicações traumáticas (800-959) e Intoxicações e efeitos tóxicos (960-989), ambos com 15 (34,0\%) casos e Outros transtornos do SNC (340-349) e Sintomas, sinais e afecções mal definidas (780-789), tais como as de pele, nutrição e outras, também com números iguais a $4(9,0 \%)$ casos. As demais categorias apresentaram números inferiores, mas nem por isso deixam de ser importantes.

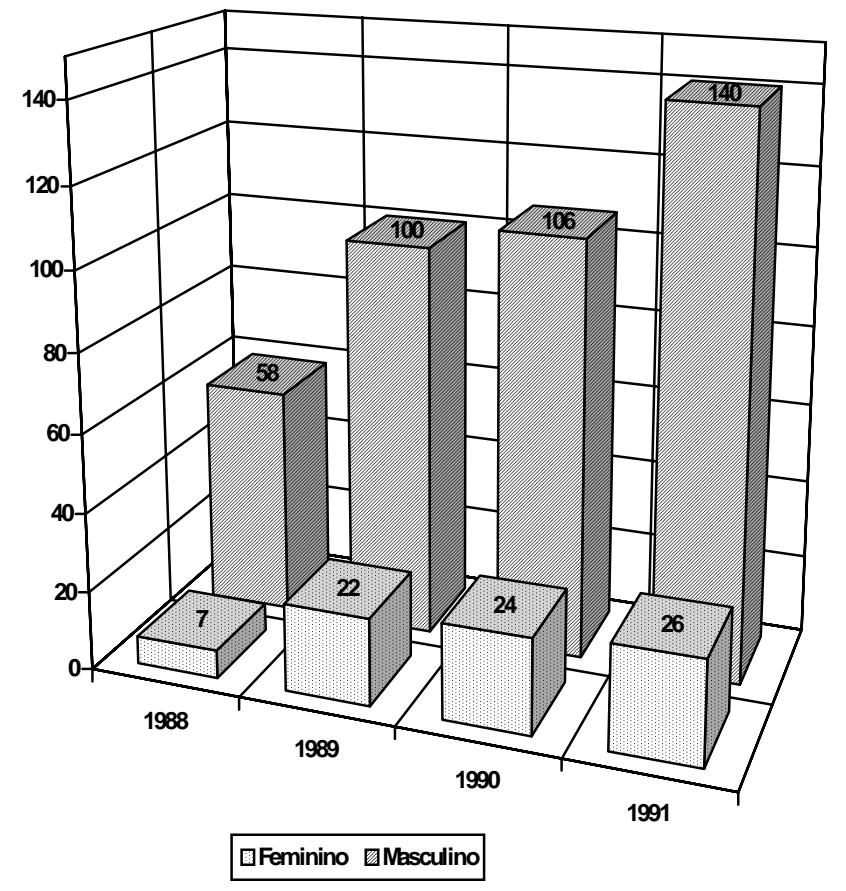

Figura 3. Distribuição dos atendimentos por sexo e ano

A Figura 3 demonstra a evidente predominância do sexo masculino, embora venha a insinuar que os diagnósticos relacionados ao álcool e associados a intercorrências das clínica médica e/ou cirúrgicas, vem aumentando também nas mulheres.

\section{DISCUSSÃO}

Segundo FORTES (1975), os setores do organismo atingidos mais severamente são: fígado, pâncreas, sistema cardiovascular, aparelho digestivo, rins, aparelho respiratório, sangue e tecido hematopoiético, aparelho reprodutor, musculatura esquelética, glândulas endócrinas e sistema nervoso central.

Outros autores tais como JORGE \& FERRAZ (1981) também revelam a associação de alterações emocionais e orgânicas nos indivíduos que ingerem abusivamente álcool, num estudo sobre o que leva um alcoólatra à internação, tendo encontrado na sua amostra, que $67,8 \%$ das primeiras internações se deve ao comprometimento a nível psicológico ou orgânico (vômitos, tremores, caimbras, desmaios, anorexia, insônia, nervosismo, agressividade, alucinações, tentativa de suicídio).

No presente estudo foi encontrado que 487 (26,5\%) dos casos de pacientes com diagnósticos relacionados ao álcool apresentaram co-morbidade com distúrbios e intercorrências de natureza orgânica e dentre as categorias diagnósticas que caracterizaram essas intercorrências, predominaram as seguintes: Outros transtornos do SNC (340-349), Lesões e complicações traumáticas (800-959), Doenças do aparelho digestivo em geral (530-537), Intoxicações e efeitos tóxicos (960-989) e por último, Sintoma, sinais e afecções mal definidas (780-789).

As três primeiras categorias diagnósticas mencionadas apareceram, com maior frequência associadas ao diagnóstico Síndrome de Dependência do Álcool (303) e embora, tenham estado presentes, também em associação com o diagnóstico Psicose Alcoólica (291), especialmente, para este quadro, a ordem de aparecimento dessas categorias diagnósticas, apresentou-se invertida.

Como informação adicional, salienta-se que no tocante ao diagnóstico Álcool (305.0), as categorias diagnósticas orgânicas não seguiram o mesmo padrão de ocorrência, sendo que apenas as Lesões e Complicações Traumáticas (800-859), manteve-se como uma das mais frequentes.

Essa frequência de associações dos distúrbios psiquiátricos relacionados ao álcool, com os quadros orgânicos evidenciados, de certa forma, já era esperada uma vez que se constituem em complicações comuns já referidas pela literatura e vivenciadas na prática profissional de quem presta assistência aos usuários de álcool (aditos ou não). Os resultados desta investigação, apenas constataram uma realidade já conhecida.

Quanto ao aumento das intercorrências das clínicas médicas e/ou cirúrgicas no sexo feminino, julgase que isso venha a ser reflexo do aumento no consumo de álcool, também nessa população, dado esse também constatado por HOCHGRAF (GREA-s/d). Entretanto, para a referida autora é discutível o aumento da prevalência de alcoolismo entre o sexo feminino, como tem sido propagado por alguns autores; no seu entender o que pode estar ocorrendo é um melhor diagnóstico da doença nessa população, devido a que as mulheres, por estarem superando a vergonha e o preconceito em relação ao seu problema, estão buscando mais os serviços especializados. 
Entretanto, é fato que apesar das mulheres começarem a beber mais tarde que os homens, apresentam-se ao tratamento com a mesma idade, o que tem levado os especialistas a pensar que a doença tem um curso mais rápido entre o sexo feminino (BLUME, 1986; JOHN, 1987; RAMOS, 1991).

De maneira geral, ficou evidente a complexidade da assistência ao alcoolista, uma vez que um número considerável de pacientes com diagnóstico relacionado ao álcool apresentaram associação com outros diagnósticos, não incluídos na área da psiquiatria, caracterizando o fato de que o alcoolismo deve ser encarado como um distúrbio grave, que atinge o indivíduo como um todo e que consequentemente, a assistência deve ser enfocada de uma maneira global, atingindo num primeiro momento ao menos suas necessidades físicas e psíquicas.

\section{CONCLUSÃO}

Esta investigação vem confirmar os achados da literatura em relação ao tema, bem como corroborar aquilo que outros estudiosos da área da saúde vem mostrando ao longo dos anos, os quais identificam o alcoolismo como um grave problema de saúde. Os resultados, embora restritos a um Setor de um hospital geral, evidenciaram que o alcoolismo extrapola a psiquiatria. Trata-se pois, de uma entidade patológica que requer cuidados de diversas especialidades médicas e cuidados multiprofissionais. Entretanto, a realidade da prática não parece estar condizente com essa necessidade observada.

As autoras partilham da concepção de JORGE (1983) de que o alcoolismo é simultaneamente uma doença (enquanto conduta individual) e uma denúncia (enquanto resultado da interação com determinado meio) e que a sociedade que reprime o álcool é a mesma que fomenta o alcoolismo. Portanto, é necessário que os profissionais de saúde percebam o paciente alcoolista, como vítima dessa contradição e busquem entendê-lo não somente em termos de alterações psíquicas, que procurem assistilo também nas suas intercorrências físicas e ajudá-lo a buscar o apoio necessário à sua reintegração social.

\section{PSYCHIATRIC DISORDERS RELATED TO ALCOHOL AND ASSOCIATED TO GENERAL CLINICAL MEDICAL DIAGNOSES AND/OR SURGICAL INTERVENTIONS IN PATIENTS ADMITTED IN A GENERAL HOSPITAL}

Through an epidemiological examination, the authors searched for co-morbidities and for psychiatric diagnoses related to alcohol. So, patients attended in a Psychiatric ward at an Emergency Service from 1988 to 1991 were investigated. The aim was to identify clinical diseases and another incidents that assault this population. The diagnoses examined were the ones related to alcohol, according to CID-9 $(291 ; 303 ; 305.0)$ and the ones associated that do not belong to psychiatry 1835 cases were observed with diagnoses related to alcohol and among them 487 (26.5\%) were concerning to the groups 291-303-305.0, which were associated to another psychiatric diagnoses. The larger frequency occurred in the groups 340-349 (Another central nervous system disorders) and 800-859 (Lesions and traumatic complications) with 80 (16.4\%) patients, respectively. Through data observation by year, authors found that among the diagnoses related to alcohol (with clinical diagnoses associated), the 303 (Alcohol dependence syndrome) was the one that had the larger number of patients with 326 (67\%) cases, followed by the 291 (Alcohol psychoses) with 117 (24\%) cases and the 305.0 (Alcohol) with 44 cases (9\%). In the period, the psychiatric diagnoses 303 and 291 appeared more frequently associated to the group of clinical diagnoses 340-349, with 55 (11,2\%) and 12 (2.4\%) cases respectively and the 305.0 had the group 800-859 with 10 (2\%) cases as the more frequent.

\section{DISTURBIOS PSIQUIÁTRICOS RELACIONADOS AL ALCOHOL, ASOCIADOS A DIAGNÓSTICOS DE CLÍNICA MÉDICA Y/O INTERVENCIONES QUIRÚRGICAS, ATENDIDOS EN UN HOSPITAL GENERAL}

Usando un formulario de notificación epidemiológica, se buscó detectar las co-morbilidades de los diagnósticos psiquiátricos relacionados con el alcohol. Para esto se investigó las atenciones realizadas en un hospital de urgencias médicas en el sector de psiquiatría, durante el periodo de 1988 a1991; teniendo como objetivo identificar cuales son las enfermedades clínicas y otros incidentes padece la población referida. Hicieron parte los diagnósticos relacionados al alcohol, conforme CID 9 (291; 303 y 305.0) y los asociados no pertenecientes a psiquiatría. En el periodo se encontró 1835 casos de diagnóstico relacionados al alcohol y de estos 487 (26,5\%) se referían a los grupos 291-303-305.0, asociados a otros no psiquiátricos. De ese total la mayor frecuencia de casos ocurrió en los grupos 340-349 (Otros trastornos del sistema nervioso central) y 800-859 
(Lesiones y complicaciones traumáticas) con 80 (16,4\%) casos atendidos respectivamente. Observándose los casos por año se constata que los diagnósticos relacionados al alcohol (con diagnósticos clínicos relacionados) y 303 (Síndrome de dependencia alcohólica) fue el que tuvo mayor número de atenciones con 326 (67\%) casos, seguido de 291 (Psicosis alcohólica) con 117 (24\%) y 305.0 (alcohol) 44 (9\%) en el periodo; tanto el diagnóstico 303 como el 291, aparecieron mas frecuentemente asociados al grupo de diagnóstico clínico 340-349, con 55 (11,2\%) y 12 (2,4\%) casos, respectivamente y el 305.0 tuvo el grupo $800-859$ con $10(2 \%)$ casos, como más frecuente.

TÉRMINOS CLAVES: alcoholismo, clínica médica y alcohol, intervenciones quirúrgicas y alcohol, psiquiatría

\section{REFERÊNCIAS BIBLIOGRÁFICAS}

01. BLUME, S. Women and alcohol :a review. JAMA, v. 256, p.1467-1470,1986.

02. BRASIL.,Ministério da Saúde. Programa Nacional de Controle dos Problemas Relacionados com o consumo de Álcool - PRONAL. Brasília: Ministério da Saúde/Disan, 1990.

03. CENTRO DE VIGILÂNCIA EPIDEMIOLÓGICA PROFESSOR ALEXANDRE VRANJAC (CVE). Internações Psiquiátricas no Estado de São Paulo. São Paulo. Secretaria da Saúde de São Paulo. 1991. 30p.

04. CERVESATO, A.C.Z. Alcoolismo: como conhecer e tratar. Jornal Psicólogos Saúde Pernambuco, v. 1, n. 2, dez. 1993.

05. CORDEIRO DE FARIAS, R.; PERNAMBUCO, F.P.; PARREIRAS, D. O alcoolismo no conceito da Organização Mundial da Saúde, 1954, 41p.(mimeo)

06. EDWARDS, G.O. Tratamento do alcoolismo. São Paulo: Martins Fontes, 1985.

07. FORTES, J.R. de A. Alcoolismo. São Paulo: Sarvier, 1975. 74p.

08. HILL, S. The disease concept of alcoholism, 16, p. 193-214, 1985.
09. HOCHGRAF, P.B. Problemática do alcoolismo e outras farmacodependências nas mulheres. GREA. Lemos. São Paulo: s/d. p. 69-78.

10. JOHN, U. Alcohol-dependent men and women in detoxification: some comparisons. A.E.R., n. 11, p. $155-157,1987$.

11. JORGE, M.R. Aspectos sociais, culturais, políticos e econômicos relacionados ao uso e abuso de bebidas alcoólicas no Brasil. Rev. Assoc. Bras. Psiquiatr., São Paulo, v.4, n.18, p.177-178, 1983.

12. JORGE, M.R.; FERRAZ, M. A percepção pelo alcoólatra do que o leva ao álcoolismo e a internação. Boletim de Psiquiatria. São Paulo, ud. XIV, n. 3, p. 132-137. set. 1981.

13. MASUR, J. A questão do alcoolismo. São Paulo: Brasiliense, 1984. 74p.

14. RAMOS, R.T. Alcoolismo feminino. In: FORTES, J.R.A; CARDO, W.N. (eds). Alcoolismo. São Paulo: Savier, 1991.

15. SCHUKIT, M. Overview: epidemilogy of alcoholism. In: SCHUCKIT, M. Alcohol pattens \& problems New Jersey: Rutgers University Press, 1985. p. 274.

16. WHO. Expert Comitee on Drug Dependence. Geneve, WHO, 1970. (Technical Report Series, n. 437). 